Pacific Journal of Mathematic 


\title{
BOUNDEDNESS IN TOPOLOGICAL RINGS
}

\author{
EDWIN WEISS
}

Introduction. The purpose of this note is to dispose of certain preliminaries (and of some peripheral remarks) in the direction of a structure theory of the Wedderburn-Artin-Jacobson type for a rather restricted class of topological rings-namely, bounded ones. The notion of boundedness, which may be looked on as an algebraic analogue of compactness, was introduced by Shafarevich [6] and later considered by Kaplansky [3]. It is not unexpected that in an algebraic approach to the study of topological rings the concept of boundedness should prove fruitful; where, by an algebraic approach is meant one in which the use of deep topological facts is avoided-thus, for example, we shall not use any results about the structure of locally compact groups, since such results depend on Haar measure, the Peter-Weyl theorem and Pontrjagin duality.

Since the study of the radical is one of the foundation stones of the classical structure theory of rings, and in view of our self-imposed restrictions on available techniques, it is natural to attempt to extend the notion of radical in such a way as to take the topology of the ring into account. Such an attempt is the primary concern of this note. The proofs will often be merely slight extensions of the standard ones for discrete rings.

1. Definitions and preliminaries. As usual, (see, for example, [3]) by a topological ring we mean a set $R$ which is a ring and a Hausdorff space and such that the mappings $(x, y) \rightarrow x-y$ and $(x, y) \rightarrow x y$ of $R \times R \rightarrow R$ are both continuous. A subset $S$ of $R$ is left bounded if for any neighborhood $U$ of 0 there exists a neighborhood $V$ of 0 ( $V$ depends on $U$ ) such that $V \cdot S \subset U$, where $V \cdot S=\{x y \mid x \in V, y \in S\}$. Right boundedness is defined in an analogous way. We say that $S$ is bounded if it is both left and right bounded. It is clear that a subset $S$ of $R$ is bounded if and only if, for any neighborhood $U$ of 0 , there exists a neighborhood $V$ of 0 such that $V \cdot S \cdot V \subset U$. If the set $R$ itself is bounded, we say that $R$ is a bounded ring.

Let $M$ be a left $R$-module; $M$ is called a topological left $R$-module when: $R$ is a topological ring, $M$ is a topological group (this includes Hausdorff), and the map $(\alpha, x) \rightarrow \alpha x$ of $R \times M \rightarrow M$ is continuous. Similarly, the notion of topological right $R$-module is defined. Since there is no essential distinction between right and left, we shall usually state things only for topological left $R$-modules.

Received November 26, 1954. This paper was written while the author was an NSF post-doctoral fellow. 
Definition 1. Let $M$ be a topological left $R$-module. A subset $S$ of $M$ is $R$-bounded if for every neighborhood $U$ of 0 in $M$, there exists a neighborhood $V$ of 0 in $R$ such that $V \cdot S \subset U$. A subset $S$ of $R$ is $M$-bounded is for every neighborhood $U$ of 0 in $M$ there exists a neighborhood $V$ of 0 in $M$ such that $S \cdot V \subset U$. If $M$ is an $R$-bounded set, we say that $M$ is $R$-bounded; and similarly we define $R$ is $M$-bounded.

Some of the elementary properties ${ }^{1}$ of topological left $R$-modules are the following:

(1) If for each $\alpha, M_{\alpha}$ is a topological left $R$-module, then the direct product $M=\Pi_{\alpha} M_{\alpha}$ becomes a topological left $R$-module in a natural way; moreover, if each $M_{\alpha}$ is R-bounded, then $M$ is $R$-bounded.

(2) Any finite set in a topological left $R$-module $M$ is $R$-bounded.

(3) Any subset of an $R$-bounded set is $R$-bounded.

(4) The union of a finite number of $R$-bounded sets is $R$-bounded.

(5) The closure of an $R$-bounded set is $R$-bounded.

(6) Every compact set in $M$ is $R$-bounded.

(7) If $S$ and $T$ are both $R$-bounded, then $S+T$ is $R$-bounded.

(8) If $M$ is discrete, then $R$ is $M$-bounded.

(9) If $R$ is discrete, then $M$ is $R$-bounded.

(10) Any convergent sequence $\left\{a_{i}\right\}$ in $M$ is $R$-bounded.

(11) If $T$ is an $R$-bounded subset of $M$, and $S$ is a left bounded subset of $R$, then $S \cdot T$ is an $R$-bounded subset of $M$.

It should be noted that the above statements are true when $R$ and $M$ are interchanged-provided the new statement has meaning. The proofs are rather trivial; however, for the convenience of the reader, we remark that the proof of (6) is essentially the same as that of [3, Lemma 10], and we also give the proof of (5). Let $\mathscr{C}$ denote the set of all neighborhoods of 0 in $M$, and $\mathscr{R}$ denote the set of all neighborhoods of 0 in $R$. Suppose that $S$ is an $R$-bounded subset of $M$, and let $U \in \mathscr{L}$; there exists a $U^{\prime} \in \mathscr{M}$ such that $U^{\prime}+U^{\prime} \subset U$; by boundedness, there exists $V^{\prime} \in \mathscr{R}$ such that $V^{\prime} \cdot S \subset U^{\prime}$; finally, there exist $V^{\prime \prime} \in \mathscr{R}$ and $W^{\prime} \in \mathscr{M}$ such that $V^{\prime \prime}$. $W^{\prime} \subset U^{\prime}$. Since $\bar{S}=\bigcap_{W \in \mathscr{M}}(S+W)$, for $V=V^{\prime} \cap V^{\prime \prime}$ we have: $V \cdot \bar{S} \subset V$. $\left(S+W^{\prime}\right) \subset V \cdot S+V \cdot W^{\prime} \subset U$.

Suppose that $R$ is a topological ring, then $R^{+}$, the additive group of $R$, may be viewed as either a topological left $R$-module or as a topological right $R$-module. Thus, to show that a subset $S$ of $R$ is

${ }^{1}$ Most of these are merely translations to the module case of statements to be found in [3, p. 161]. 
bounded, is equivalent to showing that $S$ is an $R$-bounded subset of $R^{+}$ in the left and right module cases. In particular, the properties listed above are valid for topological rings-for example, a compact subset of a topological ring is bounded. ${ }^{2}$

Since in the sequel, we shall be concerned mostly with the study of bounded rings, it is perhaps of interest to determine what applicability our results can have to normed algebras. The answer is given in the following:

THEOREM 1. Let $R$ be a topological ring, and suppose that $R$ is a locally convex topological linear space over $F$ (the reals or complexes). Then, for any bounded additive subgroup $B$ of $R$, we have $R \cdot B=(0)$.

Proof. Suppose that $B$ is bounded as a subset of $R$. Let $f$ be a fixed continuous linear functional on $R^{+}$, and let $I_{f}=\{a \in R \mid f(a \cdot B)=(0)\}$; so $I_{f} \neq \phi . \quad I_{f}$ is a subgroup of $R^{+}$, so that if we show that it is open then it is also closed, and therefore, $I_{f}=R$ since $R$ is connected. From this it follows that $f(R \cdot B)=(0)$ for all continuous linear functionals $f$; hence, by the Hahn-Banach theorem, $R \cdot B=(0)$.

It remains to show that $I_{f}$ is open. Let $U$ be a neighborhood of 0 in $R^{+}$such that $f(U)<\frac{1}{2}$; by boundedness of $B$, there exists $V \in \mathscr{R}$ such that $V \cdot B \subset U$. It suffices to show that $V \subset I_{f}$. For every $x \in V$ and every integer $n$, we have $n x B=x(n B) \subset x B \subset U$; hence, for every $b \in B$ and every integer $n, f(n x b)=n f(x b)<\frac{1}{2}$; therefore, $f(x B)=(0)$ and $V \subset I_{\rho}$.

From the theorem we have immediately:

CoRollary 1. Let $R$ be a normed algebra, then $R$ cannot be a bounded topological ring unless its multiplication is trivial.

It may be remarked that essentially the same proof as that given for Theorem 1 yields:

Proposition 1. Let $M$ be a locally convex topological linear space over $F$ (reals or complexes), then $M$ contains no $F$-bounded subgroups other than (0).

\section{Topological right quasi-regularity.}

Definition 2. An element $x$ of a topological ring $R$ is topologically right quasi-regular ${ }^{3}$ if for any neighborhood $U$ of 0 in $R$, there exists an element $y$ (depending on $U$ ) such that $x \circ y \in U$, where $x \circ y=x+x y+y$.

${ }^{2}$ This is precisely [3, Lemma 10$]$.

3 A generalization of the notion of right quasi-regularity, [2, Def. 1]. 
An ideal $I$ of $R$ is topologically right quasi-regular if every element of $I$ is topologically right quasi regular. We shall abbreviate both of these by top rqr.

Our immediate aim is to prove:

Theorem 2. Let $R$ be a left bounded topological ring, and let $N$ denote the sum of all top rqr right ideals. Then $N$ is a closed two-sided ideal of $R$.

The proof will be an immediate corollary of the lemmas which follow :

Lemma 1. Let $I$ be a top rqr right ideal in the left bounded ring $R$; if $x \in I$ and if $y$ is any top rqr element of $R$, then $x+y$ is top rqr.

Proof. Suppose we are given $U \in \mathscr{R}$; there exists $W \in \mathscr{R}$ such that $W+W+W \subset U$; and, by left-boundedness, there exists a $V \in \mathscr{R}$ such that $V \subset W$ and $V \cdot R \subset W$; also, since $y$ is top rqr, there exists $y^{\prime} \in R$ with $y \circ y^{\prime} \in V$. We have then:

$$
(x+y) \circ y^{\prime}=x+x y^{\prime}+y+y y^{\prime}+y^{\prime}=x+x y^{\prime}+y \circ y^{\prime} .
$$

Finally, since $x+x y^{\prime} \in I$, there exists $z \in R$ such that $\left(x+x y^{\prime}\right) \circ z \in V$; consequently,

$$
(x+y) \circ y^{\prime} \circ z \in\left(x+x y^{\prime}\right) \circ z+V+V \cdot z \subset V+V+W \subset U .
$$

COROLlaRY 2. In a left bounded ring, the sum of two top rqr right ideals is a top rqr right ideal.

From this corollary, it follows immediately that $N$ is a top rqr right ideal.

Lemma 2. Let $S$ be any set of top rqr elements in the left bounded ring $R$. Then any element $x$ in the closure of $S$ is top rqr.

Proof. Given any $U \in \mathscr{R}$, there exists $V \in \mathscr{R}$ such that $V+V+$ $V \subset U$; then there is a $W \in \mathscr{R}$ such that: $W=-W, W \cdot R \subset V$ and $W \subset V$. Since $x+W$ is a neighborhood of $x$, there exists $y \in S$ with $y \in x+W$; also, there is a $y^{\prime} \in R$, such that $y \circ y^{\prime} \in W$. From the identity,

$$
x \circ y^{\prime}=y \circ y^{\prime}+(x-y)+(x-y) y^{\prime},
$$

it follows that

$$
x \circ y^{\prime} \in W+W+W \cdot R \subset V+V+V \subset U .
$$


The lemma clearly implies:

Corollary 3. In a left bounded topological ring $R$, both $N$ and the set of all top rqu elements are closed sets.

Lemma 3. In a left bounded ring $R, z \in N$ if and only if $\{n z+z a\}$ is top rqr for all integers $n$ and all $a \in R$.

Proof. Trivial, since $N$ is a top ror right idea land $\{n z+z a\}$ is the smallest right ideal containing $z$.

Lemma 4. In a left bounded ring $k$, if the element $z b$ is top rqr, then so is bz.

Pron. Given $U \in \mathscr{R}$, there exists $W \in \mathscr{R}$ such that $(-b) \cdot W \subset U$; and, by boundedness, there is a $V \in \mathscr{R}$ with $V \cdot R \subset W$; hence, $(-b)$. $V \cdot R \subset U$. Now, there exists $w \in R$ such that $(z b) \circ w \in V$; therefore, $(b z) \circ(-b z-b w z)=b z-b z-b w z-b z b z-b z b w z=-b(z b \circ w) z \in(-b) \cdot V \cdot R \subset U$.

Corollary 4. In a left bounded ring $R, N$ is a left ideal. This also completes the proof of Theorem 2 .

\section{Some properties of $N$.}

Proposition 2. Let $R$ be a left bounded ring, and denote its Jacobson radical by $J$, then $J \subset N$; moreover, if $R$ is discrete or compact, $J=N$.

Proof. The inclusion $J \subset N$ requires no boundedness assumption. The discrete case is trivial; while the statement for the compact case follows from:

LeMMA 5. In a compaci ring $\Omega, x$ is top $r q r \Leftrightarrow x$ is $r q r$.

Proof. $x$ is top rqr implies that $0 \in \overline{x \circ R}$; but $x \circ R$ is the continuous image of a compact set, hence is closed; thus, $0 \in x \circ R$ which means that $x$ is rqr.

An example of a situation in which $J \neq N$ is the following: Let $P$ be the ring of $p$-adic integers, $R$ the ring of row-finite matrices over $P$. Topologizing $R$ with the finite topology, namely, by taking as neighborhoods of 0 all matrices with first $n$ rows all 0 , gives $R$ the

See $[2$, p. 303!. 
structure of a left bounded ring. It is known ${ }^{5}$ that in this case $J$ is not closed; hence $J \neq N$.

Proposition 3. Let $R$ be a left bounded ring, then $N$ contains no idempotents other than 0 .

Proof. Let $e \in N$ be an idempotent. Given any $U \in \mathscr{R}$, there exists $V \in \mathscr{R}$ such that $e V \subset U$. Since $-e \in N$, it is top rqr, hence there exists $y \in R$ with $(-e) \circ y \in V$. Thus, $-e-e y+y \in V$ and $-e-e y+e y \in e V \subset U$. This means that $-e \in U$ for every $U \in \mathscr{R}$, therefore $e=0$.

For every element $a \in R$, we denote by $Z_{a}$ the right ideal of $R$ composed of all the elements $\{x+a x\}$ where $x$ runs over $R$.

Proposition 4. In any topological ring $R, Z_{a}$ is dense in $R$ if and only if a is top rqr.

Proof. Suppose that $\bar{Z}_{a}=R$; then, in particular, for any $V \in \mathscr{R}$ there exists an $x \in R$ such that $x+a x \in-a+V$; that is, there exists $x \in R$ with $a \circ x \in V$. Conversely, suppose that $a$ is top rqr ; then given any $U \in \mathscr{R}$ there exists $y \in R$ with $a \circ y \in U$; this means that $a \in-y+$ $a(-y)+U \subset Z_{a}+U$. Therefore, $a \in \bar{Z}_{a}$ and $\bar{Z}_{a}=R$.

Lemma 6. In any topological ring $R$, if a subsequence of $\left\{x^{n}\right\}$ approaches 0 , then $x$ is top rqr.

Proof. Clearly, for every positive integer $i$, we have: $x^{i}+x^{i+1} \in Z_{x}$. For even integers $2 n$, this gives: $x^{2 n}+x \in Z_{x}$, while for odd integers $2 n+1$ we have: $x^{2 n+1}-x \in Z_{x}$. Since a subsequence of either $\left\{x^{2 n}\right\}$ or $\left\{x^{2 n+1}\right\}$ approaches 0 , we see that $x \in \bar{Z}_{x}$; so $\bar{Z}_{x}=R$ and $x$ is top rqr.

Definition 3. Let $R$ be a topological ring; we say that $x \in R$ is topologically nilpotent (top nilpotent) if $x^{n} \rightarrow 0$. An ideal is top nil if every element is top nilpotent. An ideal $I$ is top nilpotent if given $U \in \mathscr{R}$ there exists an integer $m$ with $I^{n} \subset U$ for $n \geqq m$.

Lemma 6 thus implies:

CoRollary 5. In a left bounded ring, $N$ contains all top nil right ideals.

Lemma 7. Let $S$ denote the set of all top rqr elements of $R$. 
(1) If $R$ is left bounded then:

$$
b \in b(-S) \Rightarrow b=0 ;
$$

(2) Let $M$ be a topological right $R$-module such that $R$ is $M$ bounded, then if $b \in M$ is such that $b \in \overline{b(-S)}$ then $b=0$.

Proof. We prove (1) only, the proof of (2) being identical. To be more explicit, we must show that, if $b \in R$ satisfies the following condition: for every $U \in \mathscr{R}$ there is an $x_{U} \in R$ with $-x_{U}$ top rqr and such that $b x_{U}=b+\mu$, where $\mu \in U$, then $b=0$.

Now, starting with $U$, there exists a symmetric $U^{\prime} \in \mathscr{R}$ such that $U^{\prime} \cdot R \subset U$ and $U^{\prime}+U^{\prime}+U^{\prime} \subset U$; and, there is a symmetric $W \in \mathscr{R}$ with $W \subset U^{\prime}$ and $W \cdot R \subset U^{\prime}$; finally, there is a symmetric $V \in \mathscr{R}$ with $b V \subset W$. By hypothesis, we have:

$$
b x_{W}-b-w=0 \quad \text { for some } w \in W \text {; }
$$

and since $-x_{W}$ is top rqr, there exists $y \in R$ with $\left(-x_{W}\right) \circ y \in V$; that is, $-x_{W}+y-x_{W} y \in V$. From (i) we have:

$$
b x_{w} y-b y-w y=0 \text {. }
$$

Adding, (i) and (ii) yield $b\left(x_{W} y+x_{W}-y\right)-b-w-w y=0$. Hence $b=b\left(x_{W} y+\right.$ $\left.x_{W}-y\right)-w-w y \in b V+W+W \cdot R \subset U$. Thus, $b \in U$ for every $U \in \mathscr{R}$, and $b=0$.

It may be remarked that Lemma 7 is important for applications. It is decisive for the proof of the next theorem. The module formulation will be needed in the consideration of irreducible rings of endomorphisms.

TheOREm 3. Let $R$ be a left bounded ring with descending chain condition on closed right ideals, then $N$ is algebraically nilpotent.

Proof. Consider: $N=\bar{N} \supset \bar{N}^{2} \supset \bar{N}^{3} \supset \cdots \supset \bar{N}^{n} \supset \cdots$. This is a descending chain of closed right ideals; hence, for some integer $n$, $\overline{N^{n}}=\overline{N^{k}}$ for all $k \geqq n$. We show that $M=\overline{N^{n}}=(0)$. Note first that: $N^{2 n} \subset M^{2} \subset M$ and that since $\overline{N^{2 n}}=M$, we have: $\overline{M^{2}}=M$. Suppose $M \neq(0)$; then since $M^{2}$ is dense in $M, M^{2} \neq(0)$. Let $\mathscr{S}$ be the collection of all closed right ideals $I$ satisfying the two conditions: $I \subset M$ and $I \cdot M \neq(0) . \quad \mathscr{S}$ is nonempty as $M \in \mathscr{S}$. Let $\tilde{I} \in \mathscr{S}$ be a minimal element. Since $\tilde{I} M \neq(0)$ there exists $b \in \tilde{I}, b \neq 0$ such that $b M \neq(0)$. Now $\overline{b M} \subset M$ and $\overline{b M} M \supset b M^{2} \neq(0)$, since $b M^{2}=(0) \Rightarrow b \overline{M^{2}}=(0) \Rightarrow b M=(0)$. Hence, $\overline{b M} \in \mathscr{S}$ and $\overline{b M} \subset \tilde{I}$; thus, by minimality of $\tilde{I}, \overline{b M}=\tilde{I}$. Therefore, the 
conditions of Lemma 7 are satisfied, which implies that $b=0$, a contradiction. Hence $M=(0)$.

COROLLARY 6. In a left bounded ring with descending chain condition on closed right ideals, every algebraically nil ideal is algebraically nilpotent; in fact, every top nil right ideal is algebraically nilpotent.

The following may be considered as a slight generalization of a theorem of Kaplansky. ${ }^{6}$

THEOREM 4. Let $R$ be a left bounded dual ring with no algebraically nilpotent ideals, then $N=(0)$.

Proof. $N$ is closed; hence, by [4, Theorem 2] $N$ is also a dual ring. Take any $x \in N$; since in the dual ring $N, x \in \overline{x N}$, we have $x=x y_{\varepsilon}+\varepsilon$ with $\varepsilon$ arbitrarily close to 0 in $N$. Now, $y_{\varepsilon} \in N$, so $-y_{\varepsilon}$ is top rqr ; hence, given $U \in \mathscr{R}$ there exists $z_{\sigma} \in R$ with $\left(-y_{\varepsilon}\right) \circ z_{U}=u \in U$. Therefore, $x=\left(x-x y_{\varepsilon}\right)\left(1+z_{U}\right)-x u$; and thus $x=\varepsilon+\varepsilon z_{U}-x u$. Hence $x=0$, because the right side can be made as near to 0 as desired.

4. Miscellany. Kaplansky ${ }^{7}$ has defined the notion of $Q_{r}$ ring. We extend this somewhat to the following.

Definition 4. A topological ring $R$ is $L Q_{r}$ if the set of all rqr elements contains a neighborhood of 0 . We say that $R$ is $T Q_{r}$ if the set $S$ of all top rqr elements is open, and that $R$ is $L T Q_{r}$ if $S$ contains a neighborhood of 0 .

It is known ${ }^{8}$ that in any topological ring, $L Q_{r}$ and $Q_{r}$ are equivalent; we now show:

Proposition 5. Let $R$ be a left bounded ring, then $R$ is $L T Q_{r}$ if and only if it is $T Q_{r}$.

Proof. Suppose that $R$ is $L T Q_{r}$, and let $x$ be any element of $S, U$ any element of $\mathscr{R}$, and $O_{r}$ a neighborhood of 0 all elements of which are top rqr. There then exist: $W \in \mathscr{R}$ with $W+W+W \subset U$ and $V \in \mathscr{R}$ such that $V \subset W$ and $V \cdot R \subset W$. Next, we have $y \in R$ with $x \circ y \in V$, and $V^{\prime} \in \mathscr{R}$ such that $V^{\prime}+V^{\prime} \cdot R \subset O_{r}$. Thus, for any $a \in V^{\prime}, a+a y$ is top rqr; so there exists $z \in R$ such that $(a+a y) \circ z \in V$. From the identity:

6 See [4, Theorem 3].

7 [3, p. 154].

8 [3, Lemma 2]. 


$$
(a+x) \circ y \circ z=(a+a y) \circ z+x \circ y+(x \circ y) z
$$

it follows that

$$
(a+x) \circ y \circ z \in V+V+W \subset U ;
$$

so that any element of $x+V^{\prime}$ is top rqr. Hence, $R$ is $T Q_{r}$ and the proof is complete.

Proposition 6. Let $R$ be a left bounded $L T Q_{r}$ ring, then in $R^{*}=$ $R / N, N^{*}=(0)$.

Proof. The natural map $\pi: R \rightarrow R / N$ is continuous and open; also, $N^{*}$ is a closed two sided ideal of $R^{*}$ of form $M / N$ where $M$ is a closed two sided ideal of $R$, containing $N$. To show $N^{*}=(0)$, it suffices to show that any $x \in M$ is top rqr. Given any $U \in \mathscr{R}$, let $O_{r}$ be a neighborhood of 0 with all elements top rqr; since $\pi(x)$ is top rqr, there exists $y \in R$ with $\pi(x) \circ \pi(y) \in \pi\left(O_{r}\right)$. Hence, $x \circ y \in O_{r}+N$ and $x \circ y$ is top rqr ; therefore, $x$ is top rqr.

Proposition 7. Let $R$ be a left bounded $L T Q_{r}$ ring, then:

(1) $z \in N \Leftrightarrow R z R \subset N$;

(2) $z \in N \Leftrightarrow z a$ is top rqr for every $a \in R$.

Proof. The standard one goes through; see for example, [2, Corollary to Theorem 5].

Lemma 8. Let $R$ be a left bounded LTQ ring, then $N$ is open.

Proof. Let $O_{r}$ be as above, then there is a $V \in \mathscr{R}$ with $V \cdot R \subset O_{r}$. Therefore, given $x \in V$, we have $x a$ is top rqr for every $a \in R$; hence $x \in N$. Thus $V \subset N$ and $N$ is open.

CoRollary 7. A left bounded $L T Q_{r}$ ring with $N=(0)$ is discrete.

Corollary 8. A compact, semi-simple $L T Q_{r}$ ring is finite.

Proposition 8. Let $R$ be a locally compact left bounded ring satisfying the 2nd axiom of countability, then: $R$ is $L T Q_{r} \Leftrightarrow R$ contains a neighborhood $W$ of 0 all of whose elements are top nilpotent.

Proof. If such a neighborhood $W$ exists, every element of $W$ is top rqr by a previous lemma.

For the converse, let $U$ be a compact neighborhood of 0 . There

9 Meaning that $N=J=(0)$. 
exists $W \in \mathscr{R}$ such that $W \cdot R \subset U$; hence, for $x \in W$, we have $x^{n} \in U$ for every positive integer $n$. Thus, $\left\{x^{n}\right\}$ has a limit point $a \in U=\bar{U}$, and there exists a subsequence $\left\{x^{n} i\right\}$ such that $x^{n} i \rightarrow a$. Then a subsequence of $\left\{x^{n} i+1^{-n} i\right\}$ approaches some $y \in U$. Clearly $a y=a$. Now, $U$ can be taken symmetric and small enough so that all its elements are top rqr. In particular, $-y$ is top rqr; so that for any $V \in \mathscr{R}$ there exists $z_{V}$ such that $(-y) \circ z_{V} \in V^{\prime}$ where $V^{\prime} \in \mathscr{R}$ is such that $a V^{\prime} \subset V$. Thus $-y+z_{V}-y z_{V} \in V^{\prime}$ and $-a y-a z_{V}-a y z_{V} \in a V^{\prime} \subset V$, that is : $-a y \in V$ for every $V \in \mathscr{R}$. Hence, $a y=0=a$ and every element of $W$ is top nilpotent.

The definition of a regular ring ${ }^{10}$ is standard; we generalize slightly to :

DEFINITION 5. A topological ring $R$ is topologically regular if for any $a \in R, a \in \overline{a R a}$; that is, for any $U \in \mathscr{R}$ there exists $v_{U} \in R$ with $a v_{U} a=a+u, u \in U$.

Proposition 9. In a lefi bounded topologically regular ring, $N=(0)$.

Proof. Given any $a \in R$ and $U \in \mathscr{R}$. If $a \in N$, we have: $a v_{U} a=a+u_{1}$, with $u_{1} \in U$. Also, since $-v_{V} a$ is top rqr, there exists $y_{U}$ such that $\left(-v_{U} a\right) \circ y_{U}=u_{2} \in U$. It follows that: $-a v_{U} a+a y_{U}-a v_{U} a y_{U}=a u_{2}$, and therefore: $-a-u_{1}+a y_{U}-a y_{U}-u_{1} y_{U}=a u_{2}$, that is: $-a=u_{1}+u_{1} y_{U}+a u_{2}$. Now, by a standard use of left boundedness, the right side can be made as small as desired.

\section{REFERENCES}

1. J. Braconnier, Sur les modules localements compacts, C. R. Acad. Sci. Paris, 222 (1946), 527-529.

2. N. Jacobson, The radical and semi-simplicity for arbitrary rings, Amer. J. Math., 67 (1945), 300-320.

3. I. Kaplansky, Topological rings, Amer. J. Math., 69 (1947), 153-182.

4. _ Dual rings, Ann. of Math., 49 (1948), 689-701.

5. _ Topological rings, Bull. Amer. Math. Soc., 54 (1948), 809-826.

6. I. Shafarevich, On the normalizability of topological fields, Comptes Rendus URSS (Doklady), 40 (1943), 133-135.

7. J. von Neumann, On regular rings, Proc. Nat. Acad. Sci., 22 (1936), 707-713.

INSTITUTE FOR ADVANCED STUDY AND

PRINCETON UNIVERSITY 


\section{PACIFIC JOURNAL OF MATHEMATICS}

\section{EDITORS}

H. L. Royden

Stanford University

Stanford, California

E. HewitT

University of Washington

Seattle 5 , Washington
R. P. Dilworth

California Institute of Technology Pasadena 4, California

E. G. Straus

University of California

Los Angeles 24, California

\section{ASSOCIATE EDITORS}

E. F. BECKENBACH

C. E. BURGESS

H. BUSEMANN

H. FEDERER

\author{
M. HALL \\ P. R. HALMOS \\ V. GANAPATHY IYER \\ R. D. JAMES
}

M. S. KNEBELMAN

I. NIVEN

T. G. OSTROM

M. M. SCHIFFER
J. J. STOKER

G. SZEKERES

F. WOLF

K. YOSIDA

\section{SUPPORTING INSTITUTIONS}

UNIVERSITY OF BRITISH COLUMBIA

CALIFORNIA INSTITUTE OF TECHNOLOGY

UNIVERSITY OF CALIFORNIA

MONTANA STATE UNIVERSITY

UNIVERSITY OF NEVADA

OREGON STATE COLLEGE

UNIVERSITY OF OREGON

UNIVERSITY OF SOUTHERN CALIFORNIA
STANFORD UNIVERSITY

UNIVERSITY OF UTAH

WASHINGTON STATE COLLEGE

UNIVERSITY OF WASHINGTON

AMERICAN MATHEMATICAL SOCIETY CALIFORNIA RESEARCH CORPORATION HUGHES AIRCRAFT COMPANY 


\section{Pacific Journal of Mathematics}

\section{Vol. 6, No. $1 \quad$ November, 1956}

David Blackwell, An analog of the minimax theorem for vector payoffs..... 1

L. W. Cohen, A non-archimedian measure in the space of real

sequences ..................................... 9

George Bernard Dantzig, Constructive proof of the Min-Max theorem ..... 25

Jim Douglas, On the numerical integration of quasilinear parabolic

differential equations ............................... 35

James Michael Gardner Fell, A note on abstract measure ............. 43

Isidore Isaac Hirschman, Jr., A note on orthogonal systems . . . . . . . . . . 47

Frank Harary, On the number of dissimilar line-subgraphs of a given

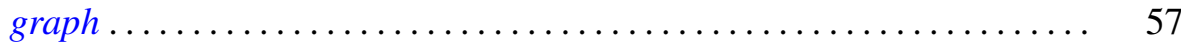

Newton Seymour Hawley, Complex bundles with Abelian group......... 65

Alan Jerome Hoffman, Morris Newman, Ernst Gabor Straus and Olga

Taussky, On the number of absolute points of a correlation ...........

Ernst Gabor Straus and Olga Taussky, Remark on the preceding paper.

Algebraic equations satisfied by roots of natural numbers . . ........ 97

Ralph D. James, Summable trigonometric series ................. 99

Gerald R. Mac Lane, Limits of rational functions . . . . . . . . . . . . . . . 111

F. Oberhettinger, Note on the Lerch zeta function ................. 117

Gerald C. Preston, On locally compact totally disconnected Abelian groups and their character groups ........................... 121

Vikramaditya Singh and W. J. Thron, On the number of singular points, located on the unit circle, of certain functions represented by

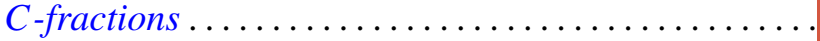

Sherman K. Stein, The symmetry function in a convex body ... 145 Edwin Weiss, Boundedness in topological rings.............

Albert Leon Whiteman, A sum connected with the series for the partition

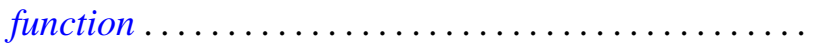

Alfred B. Willcox, Some structure theorems for a class of Banach algebras.

Joseph Lawrence Zemmer, Some remarks on p-rings and their Boolean geometry... 